\title{
DIGITAL VIDEO WATERMARKING SCHEME USING DISCRETE WAVELET TRANSFORM AND STANDARD DEVIATION
}

\author{
Souptik Sinha $^{1}$, Sneha Goswami ${ }^{2}$, Dipak kr. Kole ${ }^{3}$, Aruna Chakroborty ${ }^{4}$ \\ ${ }^{I}$ Department of Computer Science \& Engineering, St. Thomas' College of Engineering and Technology, Kolkata, India \\ ${ }^{2}$ Department of Computer Science \& Engineering, St. Thomas' College of Engineering and Technology, Kolkata, India \\ ${ }^{3}$ Department of Computer Science \& Engineering, St. Thomas' College of Engineering and Technology, Kolkata, India \\ ${ }^{4}$ Department of Computer Science \& Engineering, St. Thomas' College of Engineering and Technology, Kolkata, India
}

\begin{abstract}
Rapid development in technology has facilitated easy access to data in various domains. Security has hence become a subject of major concern. The paper brings forth a novel approach to embed a robust visible and invisible watermark into video frames, such that the authenticity of the original signal is maintained. Through Digital watermarking scheme, additional information is embedded in the video signal which protects the video from unauthorized access. This becomes instrumental in copyright owner identification. The use of Discrete Wavelet Transform (DWT) and Standard Deviation plays an important role in making this watermarking scheme resistant to various security breaches.
\end{abstract}

Keywords: Digital Watermarking, Standard Deviation and Discrete Wavelet Transform

\section{INTRODUCTION}

The advent of the Internet and the World Wide Web has revolutionized the path in which digital information is distributed. Easy access to multimedia contents and openings to device unlimited copies have envisaged the requirement for digital rights management. File-sharing technology has made it possible to copy data from a number of sources. As a result, music videos, documents are plagiarized on an unprecedented scale at optimal cost. Over the last 200 years, various countries have developed their copyright rules in a specific direction. As an example, lawmakers have reinforced the legal protections of publishers, by elevating the prices for the mass and discouraging consumption [1].

A number of watermarking techniques have been fabricated to conceal copyright markings in digital images, video, audio and other multimedia objects. The main function of digital watermarking is to embed information imperceptibly and robustly in the host digital signal. Digital watermarking is a method of copyright protection which is implemented by inserting some useful information in the original signal. An information or message is embedded in the original signal in this technique. This information is transparent to the user, both invisible and visible watermarking techniques can be used to implement this [2].

A variety of strategies have been introduced to enhance security of the media files and to verify the authenticity of the file. We have used different methods to implement these concepts. Some of the methods that resulted to be useful, depending on the quality of the extracted information, are Principal Component Analysis (PCA), Singular Value Decomposition and different works on Wavelet Transform. All the methods are used to find the least informative zone within the particular frame, where the information is to be embedded. Also methods like DCT can be used instead of DWT.

In this paper we will discuss a method which will protect a video from unauthorized access by embedding visible and invisible watermarks. An invisible watermark protects a video from being copied in the internet, but if someone takes a copy of that video using camera, then he/she will be able to claim it. To prevent this we will incorporate a visible watermark in the video which will appear in the frames dynamically depending upon the system and will be partially visible. We will use standard deviation to determine the region of less information and then we will embed the watermark. We have done the embedding based on scene change analysis. Thus this method will provide more protection to the owner's information.

The paper consists of the following sections: Section 2 deals with brief explanations of Standard Deviation and Discrete Wavelet Transform relevant to the paper, Section 3 deals with the block diagram and the proposed method, Section 4 contains the experimental results and its comparison with the previous obtained results and Section V concludes the paper. 


\section{WATERMARKING SCHEMES}

\subsection{Digital Watermarking}

A watermark is a visible or invisible data which can be integrated within a digital signal (for e.g.; video, image, audio etc. It requires no additional file header. The watermark can be identified and extracted at a later time. Watermarking helps in owner recognition and identification. It helps in determining the origin of the content. The watermark bears information about the origin, destination, ownership, transaction and copy control of the multimedia content [3].

Watermarking schemes can be either of Spatial Domain or of Transform Domain. One of the methodologies to implement watermarking in the spatial domain is to just flip the lowest order bit of chosen pixels, but this method has a disadvantage. It works efficiently only when the image is not subjected to any sort of alteration [4]. A more robust watermark can be embedded by superimposing a symbol over an area of the picture. This is achieved in transform domain and has been proved to be more robust than the ones obtained in the spatial domain. It is because in this technique, the image is inverse wavelet transformed. Hence, the watermark is dispersed irregularly over the image. This makes it difficult for the attacker to modify the image.

\subsection{Standard Deviation}

Standard Deviation is used in probability theory and statistics to measure variability. From the standard deviation we can measure how much variation exists from the expected value or mean.

Let $\mathrm{X}$ is a random variable, and then the standard deviation of $\mathrm{X}$ is

$$
\sigma_{X}=\sqrt{E\left[(X-m)^{2}\right]}
$$

\section{$\mathrm{E}=$ average expected values}

$\mathrm{m}=$ mean value of the random variable

By using standard deviation we select the region of the video frame where the minimum information lies. First we select the region of the logo size from the video frame. We then calculate the mean and standard deviation. After this the average standard deviation of that region is computed. The logo is embedded in the region of less information in the video frame.

\subsection{Discrete Wavelet Transform}

To study Discrete Wavelet Transform (DWT), first we will have to know what a wavelet is. Wavelets are functions which are generated from a function called the mother wavelet by scaling and shift in time (frequency) domain. The wavelet function is first multiplied with the original signal. The transform, which depicts a different manner of representing the former signal, is computed for every segment obtained. If the mother wavelet is denoted by $\psi(\mathrm{t})$, the other wavelets can be represented as,

$$
\Psi \mathrm{a}, \mathrm{b}(t)=\frac{1}{\sqrt{a}} \Psi\left(\frac{t-b}{a}\right)
$$

Here, $a$ and $b$ represent the parameters for scaling and shifting respectively in the time axis [5]. a corresponds to the frequency parameter and $b$ relates to the position of the wavelet function which keeps shifting in the signal. By using Wavelet Transform we can divide a signal into a set of wavelets. Two functions are required-Scaling function, which filters the lowest level of transform denoted by $\varphi_{(\mathrm{x})}$ and the other is Wavelet function which scale each level half its bandwidth

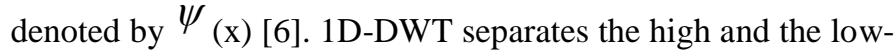
frequency portions of a signal by using filters whereas $2 \mathrm{D}$ DWT divides a signal into a set of 4 components which are LL, $\mathrm{LH}, \mathrm{HL}, \mathrm{HH}$. LL is called the lower resolution approximation component and $\mathrm{HL}$ (horizontal), LH (vertical) and $\mathrm{HH}$ (diagonal) detailed component. We will use the LL and $\mathrm{HH}$ components in our algorithm to embed watermark in video frames. DWT is more computationally efficient than other transform methods like DFT and DCT for its excellent spatialfrequency localization properties [7].

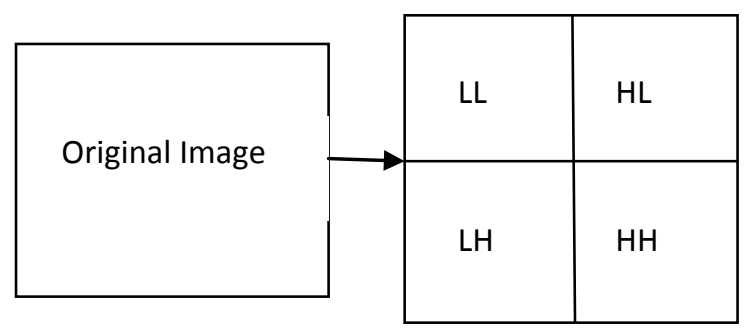

Fig 1 Applying DWT

\section{PROPOSED METHOD}

The invisible and visible watermark embedding and extraction method depends on discrete wavelet transformation followed by Standard Deviation.

For invisible watermarking we apply DWT on each video frame and then apply standard deviation to select the region where we can embed the watermark logo. For visible watermarking we will use standard deviation to select the region and then embed the logo. To select the less information region, we choose a mask which has a size equal to the size of the logo. The mask is then moved over the whole image. Standard deviation is computed over the pixel area of the mask to determine the region that is the least informative. To extract the watermark from the video, least informative region is selected and then from that region the embedded watermark is acquired. 


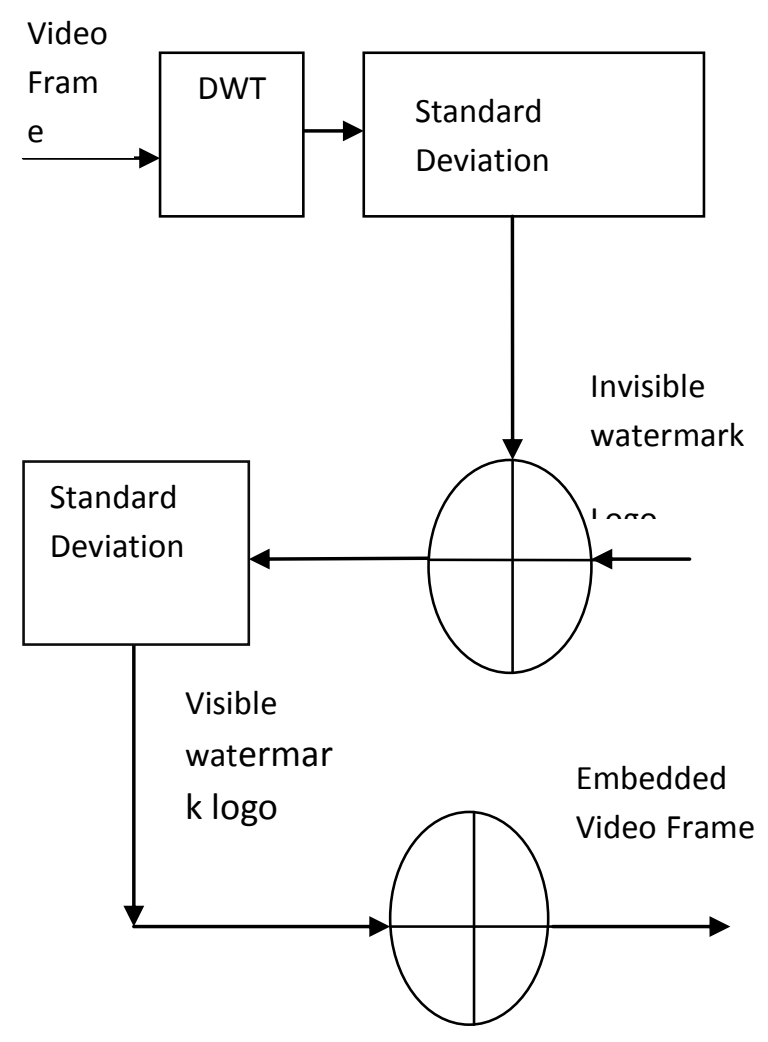

Fig 2 Block Diagram for Watermark Embedding

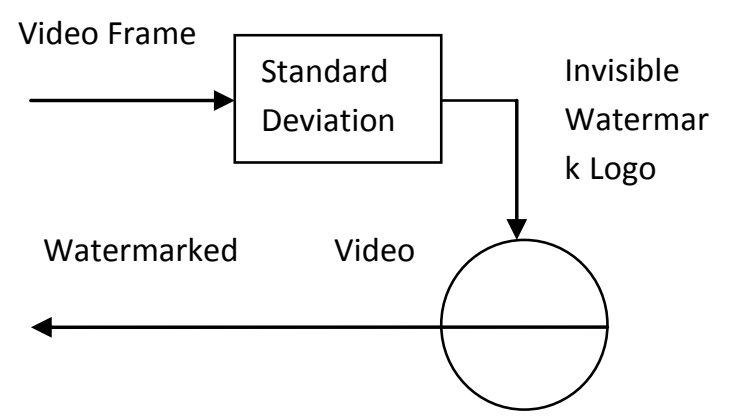

Fig 3 Block diagram for Watermark extraction

The proposed algorithm implemented in this paper is described below:

Embedding the invisible watermark:

1. Take the uncompressed video as the input,

2. Apply discrete wavelet transform into each video frames,

3. Standard deviation will be used on the output of the above transform (LL band),

4. The less informative region of the corresponding frame will be located,

5. Embed the watermark in the above located region.

Embedding the visible watermark:

1. Take the video as the input.
2. Select the less informative region by using standard deviation.

3. Embed watermark in that region.(it would be partially visible to restore the optimal quality of the video).

Extracting the invisible watermark:

1. Take watermarked video and the original video as an input.

2. Apply discrete wavelet transform on the original video frames.

3. Perform standard deviation on the video frames of the original video.

4. Perform the required computation to retrieve each pixel value of the watermark.

5. Retrieve the whole watermark from the above obtained lowest information area.

\section{EXPERIMENTAL RESULTS}

The proposed method has been applied on various images and successful results based on the quality of the watermark extracted have been received. Quality of the watermark is defined by the robustness, amount of noise in the watermark and so on. Also, it is resistant to different security breaches that may affect the authenticity of the information. Attacks likeSubtractive attack, Distortive attack, Additive attack, Filtering, and others do not have significant effects on the original information embedded in the working video.

The video used in the algorithm is an uncompressed '.avi' file. The watermark has been visibly embedded in the frames. Also an invisible watermark has been embedded with the help of DWT. The watermark used is given in the figures below.
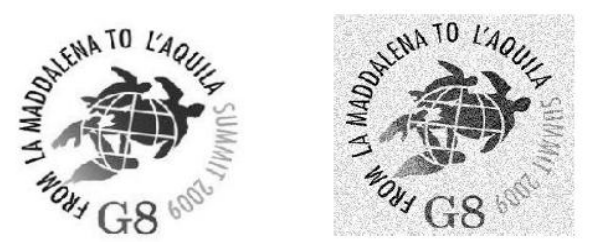

Fig 4: Watermark embedded Fig 5: Extracted Watermark

The visible watermark is not apparent in all the frames. A snapshot of one of the frames where a different watermark, is used for the frames, is partially visible and is shown in fig-6. We have implemented the algorithm in Matlab R2009a in Windows platform. 


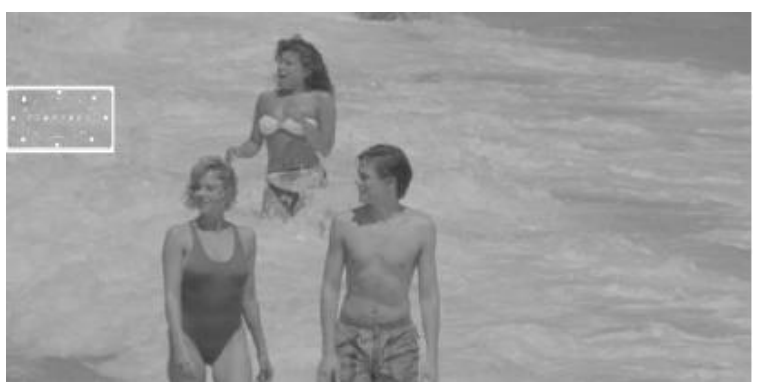

Fig 6: Watermarked Frame of the Video

\section{CONCLUSIONS}

It has been experimentally proved that this watermarking technique which utilizes DWT and standard deviation enhances the protection of the watermark and the video. The invisible watermark is embedded in the lowest frequency band (LL) which helps less degradation of video quality. In addition, it is less affected by noise and very closely resembles the original signal. Still, this technique has certain limitations within which it is restricted to deliver the optimal result. The video taken as an input is of uncompressed avi format. The whole processing is done in grayscale and the output received is also in grayscale .In other words, the proposed algorithm works best on grayscale videos and images. To improvise the above method further, we have to extend its application to all possible videos and images.

\section{FUTURE WORK}

Further modifications are possible by integrating new ideas to the proposed method. A key can be used to process the embedding. The key here is nothing but an equation. Even visual cryptography can be blended with the above method. This technique increases the security of the information and maintains inviolable authenticity.

\section{REFERENCES}

[1]. Elham Shahinfard, Shohreh Kasaei, "Digital Image Watermarking using Wavelet Transform".

[2]. T.JAYAMALAR, Dr. V. RADHA, "Survey on Digital Video Watermarking Techniques and Attacks on Watermarks", International Journal of Engineering Science and Technology, Vol. 2(12), 2010, 6963-6967.

[3]. Sourav Bhattacharya, T. Chattopadhyay and Arpan Pal, "A Survey on Different Video Watermarking Techniques and Comparative Analysis with Reference to H.264/AVC", Consumer Electronics 2006 IEEE Tenth International Symposium, 2006

[4]. Mahmoud El-Gayyar and Prof. Dr. Joachim von zur Gathen, "Watermarking Techniques Spatial Domain Digital Rights Seminar", Media Informatics, University of Bonn, Germany, May 06

[5]. Salwa A.K Mostafa, A. S. Tolba , F. M. Abdelkader, Hisham M. Elhindy, "Video Watermarking Scheme Based on
Principal Component Analysis and Wavelet Transform "IJCSNS International Journal of Computer Science and Network Security, VOL.9 No.8, August 2009

[6]. H. Liu, N. Chen, J. Huang, "A Robust DWT-Based Video Watermarking Algorithm", Proceedings of the International Conference on Signal and Image Processing Applications (ICSIPA), IEEE, pp. 352 - 356,2000. 